Dept. OF MATH/CMA. Univ. OF OSLO Statistical Research Report No 3 ISSN 0806-3842 February 2008

\title{
PRICING OF BASKET OPTIONS USING UNIVARIATE NORMAL INVERSE GAUSSIAN APPROXIMATIONS
}

\author{
FRED ESPEN BENTH AND PÅL NICOLAI HENRIKSEN
}

\begin{abstract}
In this paper we study the approximation of a sum of assets having marginal logreturns being multivariate normal inverse Gaussian distributed. We analyse the choice of a univariate exponential NIG distribution, where the approximation is based on matching of moments. Probability densities and European basket call option prices of the two-asset and univariate approximations are studied and analyzed in two cases, each case consisting of 9 scenarios of different volatilities and correlations, to assess the accuracy of the approximation. We find that the sum can be well approximated, however, failing to match the tails for some extreme parameter choices. The approximated option prices are close to the true ones, although becoming significantly underestimated for far out-of-the-money call options.
\end{abstract}

\section{INTRODUCTION}

In finance one often faces the problem of valuation of an option written on several assets, generally called a basket option. This is a problem that may be computationally demanding, since very few options have a closed-form solution for the price. In the standard multidimensional geometric Brownian motion model for the asset price dynamics, the expected value of a function of a sum of dependent lognormally distributed random variables must be calculated. Since the distribution of such sums are not known, one resorts to numerical simulations or to approximations.

It is well-known that in many cases one can approximate the sum of lognormal random variables by a lognormal random variable. By matching of the moments, there exist explicit relations between the parameters of the multivariate lognormal random variable, and the approximating univariate. We refer to Zacks and Tsokos [22], Asmussen and RojasNandayapa [2], Levy [15], Henriksen [13] and Hoedemaker [14], for extensive studies of such approximations. Obviously, having a univariate lognormal approximation is advantageous, since then we obtain a closed-form (Black \& Scholes) formula for the price. This approach is very often used when pricing basket options. When variables are negatively

\footnotetext{
Date: February 1, 2008.

Key words and phrases. Normal inverse Gaussian distribution, approximation, moment matching, basket option, simulation.

We thank Reik Börger for inspiring and interesting discussions.
} 
correlated, it turns out that the approximation is not very good, and option prices may deviate significantly from the true ones (see e.g. Henriksen [13]).

It is extensively documented in the financial literature (starting with the seminal paper of Fama [12]) showing that geometric Brownian motion is not a good model for financial asset prices. The works of Rydberg [19], Eberlein and Keller [11] and Prause [18] show that the class of generalized hyperbolic distributions provides a flexible family of distributions fitting financial data very well. In particular, the normal inverse Gaussian (NIG) distribution turns out to be very suitable for both modeling and analytical purposes, and has gained a lot of attention in the literature (see, for example, Lillestøl $[16,17]$ ). Having a multivariate asset price dynamics with NIG distributed logreturns raises the question of valid univariate approximations of its sum which can be used for efficient pricing of options. We approach this question by suggesting an approximation using the NIG distribution.

The idea to approximate the logarithm of a linear combination of exponential NIG variables with a univariate NIG was first proposed and applied to option pricing in electricity markets by Börger [8]. We extend his analysis, providing structured examples testing the validity of the approximation using different matching procedures in the bivariate case. In particular, we test the robustness of the approximations with respect to different correlation structures and tail heaviness. We discuss the approximation of a sum of two asset prices with bivariate NIG distributed logreturns, which fails for some extreme choices of parameters. The matching on log-price levels, that is, the matching of the logarithm of the sum with a univariate NIG variable, is more robust towards different assumptions, but may provide bad approximations. However, when applying our methods on options, it seems that prices are overall well approximated, also in the extreme cases. Not unexpectedly, by considering basket call options, the approximation gets worse the more out-of-the-money the option is. Interestingly, the cases where the univariate NIG approximation does not perform well is connected to situations where the bivariate NIG variables are negatively correlated. In this sense our findings are in line with those of the lognormal case.

Our results and analysis are presented as follows. In Section 2 we present necessary background material for the multivariate NIG distribution, which is used extensively in later sections. Different approximation methods based on moment matching are discussed in Section 3, where we also provide several empirical examples testing out the performance of the approximation procedures. In Section 4 we price basket call options under different distributional choices, and assess the approximation methods in terms of robustness in option prices. Finally, in the last section, we conclude.

\section{THE NORMAL INVERSE GAUSSIAN DISTRIBUtion}

Let $(\Omega, \mathcal{F}, P)$ be a complete probability space. The $d$-dimensional NIG distribution was introduced by Barndorff-Nielsen [3, 4], and has $\alpha \geq 0, \boldsymbol{\beta} \in \mathbb{R}^{d}, \boldsymbol{\mu} \in \mathbb{R}^{d}, \delta \geq 0$ and $\boldsymbol{\Delta} \in \mathbb{R}^{d \times d}$ as parameters. The matrix $\boldsymbol{\Delta}$ is positive definite with determinant 1 , and we denote by $\boldsymbol{X} \sim \operatorname{NIG}(\alpha, \boldsymbol{\beta}, \boldsymbol{\mu}, \delta, \boldsymbol{\Delta})$ for a $d$-dimensional random variable being NIG-distributed with 
these parameters. The probability density function of $\boldsymbol{X}$ is given as

$$
f_{\boldsymbol{X}}(\boldsymbol{x})=\frac{\delta}{2^{(d-1) / 2}}\left(\frac{\alpha}{\pi q(\boldsymbol{x})}\right)^{\frac{d+1}{2}} \exp (p(\mathbf{x})) K_{(d+1) / 2}(\alpha q(\boldsymbol{x}))
$$

where

$$
\begin{aligned}
& p(\boldsymbol{x})=\delta \sqrt{\alpha^{2}-\boldsymbol{\beta}^{\prime} \boldsymbol{\Delta} \boldsymbol{\beta}}+\boldsymbol{\beta}^{\prime}(\boldsymbol{x}-\boldsymbol{\mu}), \\
& q(\boldsymbol{x})=\sqrt{\delta^{2}+(\boldsymbol{x}-\boldsymbol{\mu})^{\prime} \boldsymbol{\Delta}^{-1}(\boldsymbol{x}-\boldsymbol{\mu})},
\end{aligned}
$$

and $K_{d}$ is the modified Bessel function of second kind of order $d$ (see for example [1]). The condition $\alpha^{2} \geq \boldsymbol{\beta}^{\prime} \Delta \boldsymbol{\beta}$ applies. The $\alpha$ parameter corresponds the tail-heaviness of the marginals, while $\delta$ is the scaling of the distribution. Further, $\boldsymbol{\mu}$ is the centering, and $\boldsymbol{\beta}$ controls the skewness. Finally, $\Delta$ models the dependency structure between the marginals. by

The cumulant function (defined as the logarithm of the characteristic function) is given

$$
\psi_{\boldsymbol{X}}(\boldsymbol{s})=\delta \sqrt{\alpha^{2}-\boldsymbol{\beta}^{\prime} \boldsymbol{\Delta} \boldsymbol{\beta}}-\delta \sqrt{\alpha^{2}-(\boldsymbol{\beta}+\mathrm{i} \boldsymbol{s})^{\prime} \boldsymbol{\Delta}(\boldsymbol{\beta}+\mathrm{i} \boldsymbol{s})}+\mathrm{i} \boldsymbol{s}^{\prime} \boldsymbol{\mu},
$$

with $\mathrm{i}=\sqrt{-1}$ being the imaginary unit. From this, we easily derive the mean vector and covariance matrix,

$$
\begin{aligned}
\mathrm{E}[\boldsymbol{X}] & =\boldsymbol{\mu}+\delta \boldsymbol{\Delta} \boldsymbol{\beta}\left(\alpha^{2}-\boldsymbol{\beta}^{\prime} \boldsymbol{\Delta} \boldsymbol{\beta}\right)^{-1 / 2}, \\
\operatorname{Var}[\boldsymbol{X}] & =\delta\left(\alpha^{2}-\boldsymbol{\beta}^{\prime} \boldsymbol{\Delta} \boldsymbol{\beta}\right)^{-1 / 2}\left(\boldsymbol{\Delta}+\left(\alpha^{2}-\boldsymbol{\beta}^{\prime} \boldsymbol{\Delta} \boldsymbol{\beta}\right)^{-1} \boldsymbol{\Delta} \boldsymbol{\beta} \boldsymbol{\beta}^{\prime} \boldsymbol{\Delta}\right) .
\end{aligned}
$$

We see that the relationship between $\Delta$ and the covariance matrix is nontrivial, since the covariance matrix will depend on the parameters $\alpha, \delta$ and $\boldsymbol{\beta}$ as well as $\boldsymbol{\Delta}$. In fact, the multivariate NIG-parameters can not easily be derived from univariate marginal NIG distributions and a correlation matrix. However, some approximation methods to do this can be found in Lillestøl [16].

The NIG-distribution is infinitely divisible, and we can define a $d$-dimensional Lévy process $\boldsymbol{X}(t)$ where $\boldsymbol{X}(1) \sim \operatorname{NIG}(\alpha, \boldsymbol{\beta}, \boldsymbol{\mu}, \delta, \boldsymbol{\Delta})$. We denote by $\mathcal{F}_{t}$ the filtration generated by $\boldsymbol{X}(t)$, which is assumed to satisfy the standard conditions. From the independent increment property it follows that the process is NIG-distributed at all times, with $\boldsymbol{X}(t) \sim$ $\operatorname{NIG}(\alpha, \boldsymbol{\beta}, \boldsymbol{\mu} t, \delta t, \boldsymbol{\Delta})$. In our paper, $\boldsymbol{X}$ will be the log-spot price dynamics of $d$ assets, that is, we define the price dynamics for asset $k, k=1, \ldots, d$ to be

$$
S_{k}(t)=S_{k}(0) \exp \left(X_{k}(t)\right),
$$

where $X_{k}$ is the $k$ th coordinate of $\boldsymbol{X}(t)$. Hence, the logreturns of $\boldsymbol{S}$ will be NIG-distributed. We say that a random variable $Y$ is $\log$ NIG distributed whenever $\ln Y$ is NIG distributed. Thus, $\boldsymbol{S}(t)$ is multivariate $\log$ NIG distributed.

In option pricing problems, the risk-neutral dynamics of the assets is needed. We consider here the Esscher transform of $\boldsymbol{S}(t)=\left(S_{1}(t), \ldots, S_{d}(t)\right)^{\prime}$ to derive a risk-neutral dynamics. For a parameter vector $\boldsymbol{\theta} \in \mathbb{R}^{d}$, define a probability measure $Q \sim P$ with Radon-Nikodym 
density

$$
\left.\frac{d Q}{d P}\right|_{\mathcal{F}_{t}}=\exp \left(\boldsymbol{\theta} \boldsymbol{X}(t)-\psi_{\boldsymbol{X}}(-\mathrm{i} \boldsymbol{\theta}) t\right) .
$$

We use the notation $\mathrm{E}_{Q}[\cdot]$ for the expectation under the probability $Q$. We implicitly consider only those $\boldsymbol{\theta}$ for which $\psi_{\boldsymbol{X}}(-\mathrm{i} \boldsymbol{\theta})$ is well-defined. Since the NIG-distribution has finite exponential moments up to a certain order, there exist $\boldsymbol{\theta}$ for which a probability $Q$ is defined. Following the derivations in Shiryaev [20, Ch. 7], it holds that $\boldsymbol{X}(t)$ is a Lévy process with respect to $Q$. Let us calculate the cumulant function of $\boldsymbol{X}(t)$ under $Q$.

$$
\begin{aligned}
\psi_{\boldsymbol{X}}^{Q}(\boldsymbol{s} ; \boldsymbol{\theta})= & \ln \mathrm{E}_{Q}[\exp (\mathrm{i} \boldsymbol{s} \boldsymbol{X}(1))] \\
= & \ln \mathrm{E}\left[\exp (\mathrm{i} \boldsymbol{s} \boldsymbol{X}(1)) \exp \left(\boldsymbol{\theta} \boldsymbol{X}(1)-\psi_{\boldsymbol{X}}(-\mathrm{i} \boldsymbol{\theta})\right)\right] \\
= & \mathrm{i} \boldsymbol{\mu} \boldsymbol{s}+\delta \sqrt{\alpha^{2}-(\boldsymbol{\beta}+\boldsymbol{\theta})^{\prime} \boldsymbol{\Delta}(\boldsymbol{\beta}+\boldsymbol{\theta})} \\
& \quad-\delta \sqrt{\alpha^{2}-(\boldsymbol{\beta}+\boldsymbol{\theta}+\mathrm{i} \boldsymbol{s})^{\prime} \boldsymbol{\Delta}(\boldsymbol{\beta}+\boldsymbol{\theta}+\mathrm{i} \boldsymbol{s})}
\end{aligned}
$$

Hence, $\boldsymbol{X}(1)$ is NIG-distributed under $Q$ with parameters $\alpha, \boldsymbol{\beta}+\boldsymbol{\theta}, \delta$ and $\boldsymbol{\Delta}$. Note that only the $\boldsymbol{\beta}$-parameter is changed, being simply translated by $\boldsymbol{\theta}$. In conclusion, $\boldsymbol{X}(t)$ is also a NIG-Lévy process under the probability $Q$. We have a risk-neutral dynamics if the discounted price process is a $Q$-martingale, where discounting is done using the risk-free interest rate $r>0$. Thus, we must find a $\boldsymbol{\theta}$ such that

$$
\mathrm{E}_{Q}\left[\mathrm{e}^{-r t} \boldsymbol{S}(t)\right]=\boldsymbol{S}(0)
$$

or, equivalently

$$
\psi_{\boldsymbol{X}}^{Q}\left(-\mathrm{i} \boldsymbol{e}_{k} ; \boldsymbol{\theta}\right)=1
$$

Here, $\boldsymbol{e}_{k}$ is the $k$ th unit vector in $\mathbb{R}^{d}$.

In most of the examples that we consider in this paper, we shall be concerned with the univariate and two-dimensional cases of the NIG-distribution. According to BarndorffNielsen [5] and Rydberg [19], the density function takes a simple form for dimension $d=2$,

$$
f_{\boldsymbol{X}}(\boldsymbol{x})=\frac{\delta}{2 \pi} \frac{\exp (p(\boldsymbol{x}))}{\exp (\alpha q(\boldsymbol{x}))} \frac{1+\alpha q(\boldsymbol{x})}{q(\boldsymbol{x})^{3}} .
$$

The equation in (2.5) becomes a system of 2 equations in the bivariate case, more specifically,

$$
\begin{aligned}
& r=\mu_{1}-\delta \sqrt{\alpha^{2}-\left[\beta_{1}+1+\theta_{1}, \beta_{2}+\theta_{2}\right] \Delta\left[\begin{array}{c}
\beta_{1}+1+\theta_{1} \\
\beta_{2}+\theta_{2}
\end{array}\right]} \\
& \quad+\delta \sqrt{\alpha^{2}-\left[\beta_{1}+\theta_{1}, \beta_{2}+\theta_{2}\right] \Delta\left[\begin{array}{l}
\beta_{1}+\theta_{1} \\
\beta_{2}+\theta_{2}
\end{array}\right]}, \\
& r=\mu_{2}-\delta \sqrt{\alpha^{2}-\left[\beta_{1}+\theta_{1}, \beta_{2}+1+\theta_{2}\right] \Delta\left[\begin{array}{c}
\beta_{1}+\theta_{1} \\
\beta_{2}+1+\theta_{2}
\end{array}\right]}
\end{aligned}
$$




$$
+\delta \sqrt{\alpha^{2}-\left[\beta_{1}+\theta_{1}, \beta_{2}+\theta_{2}\right] \Delta\left[\begin{array}{l}
\beta_{1}+\theta_{1} \\
\beta_{2}+\theta_{2}
\end{array}\right]} .
$$

This system of equations must be solved numerically, a problem we will come back to in Section 3.

The univariate case gives a density function

$$
f_{X}(x)=\frac{\delta \alpha}{\pi} \exp \left(\delta \sqrt{\alpha^{2}-\beta^{2}}+\beta(x-\mu)\right) \frac{K_{1}(\alpha q(x-\mu))}{q(x-\mu)} .
$$

Since it will be useful in the approximations that we consider in Section 3, we list the explicit expressions for the first four moments in the univariate case:

$$
\begin{aligned}
\mathrm{E}[X] & =\mu+\delta \frac{\beta}{\gamma}, \\
\operatorname{Var}[X] & =\delta \frac{\alpha^{2}}{\gamma^{3}}, \\
\operatorname{Skew}[X] & =3 \frac{\beta}{\alpha} \frac{1}{(\delta \gamma)^{1 / 2},} \\
\operatorname{Kurt}[X] & =3\left(1+\frac{\alpha^{2}+4 \beta^{2}}{\delta \alpha^{2} \gamma}\right),
\end{aligned}
$$

where $\gamma=\sqrt{\alpha^{2}-\beta^{2}}$.

We continue our exposition with a discussion on simulation of univariate and bivariate NIG-distributed random variables. First, consider the univariate case, which is the simpler of the two.

It is convenient to use a mean-variance mixture representation of $X \sim \operatorname{NIG}(\alpha, \beta, \mu, \delta)$ (see for example Rydberg [19]). Define $X$ through the conditional distribution

$$
X \mid Z=z \sim N(\mu+\beta z, z),
$$

where $Z \sim I G\left(\delta, \gamma^{2}\right)$, that is, inverse Gaussian distributed with parameters $\delta$ and $\gamma^{2}$. Thus, for a standard normally distributed random variable $W \sim N(0,1)$, we can write:

$$
X=\mu+\beta Z+\sqrt{Z} W .
$$

Hence, $X \sim \operatorname{NIG}(\alpha, \beta, \mu, \delta)$ can be simulated by drawing samples from standard normal and inverse Gaussian distributed variables. Methods for drawing from a standard normal variable are well-known, whilst drawing inverse Gaussian distributed samples is a bit more technical. We give a short presentation of the technique here, and refer to Rydberg [19] for the details.

Sample from $Z$ by first drawing a random variable $V$ being $\chi^{2}$-distributed with 1 degree of freedom. Define next a random variable $Y$ given by

$$
Y=\omega+\frac{\omega^{2} V}{2 \delta^{2}}-\frac{\omega}{2 \delta^{2}} \sqrt{4 \omega \delta^{2} V+\omega^{2} V^{2}}
$$


where $\omega=\delta / \sqrt{\alpha^{2}-\beta^{2}}$. Further, sample from $Z$ by drawing $U \sim \operatorname{Unif}(0,1)$ and let

$$
Z=Y \cdot \mathbf{1}\left(U \leq \frac{\omega}{\omega+Y}\right)+\frac{\omega^{2}}{Y} \cdot \mathbf{1}\left(U>\frac{\omega}{\omega+Y}\right)
$$

Thus, from the samples of $W$ and $Z$ we obtain univariate NIG samples by (2.13). In Benth, Groth and Kettler [6], a quasi-Monte Carlo method is developed based on this simulation algorithm.

We now turn our attention to the simulation of a bivariate NIG variable. Various strategies may be applied to simulate from a bivariate NIG, where we shall follow one of the approaches mentioned by Prause [18] based on a conditional sampling technique. The conditional sampling technique rests on Theorem 1, parts a) and b), in Blæsild [7], which we state here for convenience in the bivariate NIG case.

Theorem 2.1. Suppose that $\boldsymbol{X}=\left(X_{1}, X_{2}\right)^{\prime}$ is a bivariate random variable distributed according to $\operatorname{NIG}(\alpha, \boldsymbol{\beta}, \boldsymbol{\mu}, \delta, \boldsymbol{\Delta})$, where

$$
\boldsymbol{\beta}=\left[\begin{array}{l}
\beta_{1} \\
\beta_{2}
\end{array}\right], \quad \boldsymbol{\mu}=\left[\begin{array}{l}
\mu_{1} \\
\mu_{2}
\end{array}\right], \quad \boldsymbol{\Delta}=\left[\begin{array}{ll}
\Delta_{11} & \Delta_{12} \\
\Delta_{21} & \Delta_{22}
\end{array}\right] .
$$

Then one has the following

a) The distribution of $X_{1}$ is univariate $\operatorname{NIG}\left(\alpha^{*}, \beta^{*}, \mu^{*}, \delta^{*}\right)$, where $\alpha^{*}=\Delta_{11}^{-1 / 2}\left(\alpha^{2}-\right.$ $\left.\beta_{2}\left(\Delta_{22}-\Delta_{21} \Delta_{11}^{-1} \Delta_{12}\right) \beta_{2}\right)^{1 / 2}, \beta^{*}=\beta_{1}+\beta_{2} \Delta_{21} \Delta_{11}^{-1}, \mu^{*}=\mu_{1}$ and $\delta^{*}=\delta \Delta_{11}^{1 / 2}$.

b) The conditional distribution of $X_{2}$ given $X_{1}=x_{1}$ is the generalized univariate hyperbolic distribution $H(\tilde{\lambda}, \tilde{\alpha}, \tilde{\beta}, \tilde{\mu}, \tilde{\delta})$, where $\tilde{\lambda}=-1$, $\tilde{\alpha}=\alpha \Delta_{11}^{1 / 2}, \tilde{\beta}=\beta_{2}, \tilde{\mu}=$ $\mu_{2}+\left(x_{1}-\mu_{1}\right) \Delta_{11}^{-1} \Delta_{12}$ and $\tilde{\delta}=\Delta_{11}^{-1 / 2}\left(\delta^{2}+\left(x_{1}-\mu_{1}\right) \Delta_{11}^{-1}\left(x_{1}-\mu_{1}\right)\right)^{1 / 2}$.

We refer to Blæsild [7] for a definition of the generalized hyperbolic distribution. Using this theorem we can generate bivariate NIG samples by first generating $X_{1}$ from part a) by appealing to the method described above in the univariate NIG case, and then $X_{2}$ given $X_{1}=x_{1}$ from part $\mathrm{b}$ ). The univariate generalized hyperbolic variable in part b) can be generated by appealing to the representation

$$
X_{2}=\tilde{\mu}+\tilde{\beta} G+\sqrt{G} W
$$

where $W \sim N(0,1)$ and $G \sim \operatorname{GIG}\left(-1, \tilde{\delta}^{2}, \tilde{\alpha}^{2}-\tilde{\beta}^{2}\right)$, the generalized inverse Gaussian (GIG) distribution (see Blæsild [7]). We refer to Dagpunar [10] for a detailed description on the generation of samples from the GIG distribution.

We remark that the simulation algorithm can be easily extended to higher dimensions. This is done by partitioning a $d$-dimensional random variable $\boldsymbol{X}$ into two parts, a univariate and $d$-1-dimensional random variables. Next, partition the $d$-1-variables into a univariate and a $d$-2-dimensional variable, and iterate this until all the $d$ parts have dimension 1 . From Theorem 1 of Blæsild [7], all the $d-1$ last parts are generalized hyperbolically distributed, which can be simulated based on the algorithm in [10]. The exact form of each of the parts are rather technically complicated, and we refrain from stating them here. 


\section{Approximation using a univariate NiG Distribution}

In this section we consider the problem of approximating $\boldsymbol{a}^{\prime} \boldsymbol{S}(t)$ for a vector $\boldsymbol{a} \in \mathbb{R}^{d}$, where the dynamics of $\boldsymbol{S}(t)$ is given and defined in (2.3) with $\boldsymbol{X}(t)$ being a $d$-dimensional NIG-Lévy process. In general, the distribution of $\boldsymbol{a}^{\prime} \boldsymbol{S}(t)$ is not known even though we have a full description of it for each $S_{k}(t), k=1, \ldots, d$. In applications like pricing of basket options written on $\boldsymbol{a}^{\prime} \boldsymbol{S}(t)$, one must resort to numerical simulations that may become computationally time consuming in higher dimensions. In this Section we propose to approximate the logarithm of $\boldsymbol{a}^{\prime} \boldsymbol{S}(t)$ with a NIG-distribution, following the idea used frequently for the analogous case when the asset prices are modeled by a multi-dimensional geometric Brownian motion. In that case, one approximates the logarithm using a normal distribution (see Turnbull and Wakeman [21] where this was first suggested for Asian options, and Henriksen [13] for an extensive study of this approximation). Assuming that such a NIG approximation is good, the price of a basket option can be efficiently computed using Monte Carlo or transform-based methods. In this section we study the approximation extensively from a distribution point of view, whereas the pricing of options will be analysed in Section 4 .

We focus on the case $\boldsymbol{a}=(1,1)^{\prime}$, that is, the approximation of $S_{1}(t)+S_{2}(t)$ using a $\operatorname{logNIG}$ distributed random variable. Denoting by

$$
S(t)=S(0) \exp (X(t)),
$$

where $S(0)=S_{1}(0)+S_{2}(0)$ and $X(t)$ is an NIG-distributed random variable, we search for parameters $\alpha, \beta, \mu$ and $\delta$ such that $X(t) \sim \operatorname{NIG}(\alpha, \beta, \mu, \delta)$ and the four first moments of $S(t)$ match the corresponding moments of $S_{1}(t)+S_{2}(t)$. From the binomial formula, the $n$th moment of $S_{1}(t)+S_{2}(t)$ may be represented as (for $n \geq 1$ a natural number)

$$
\begin{aligned}
\mathrm{E}\left[\left(S_{1}(t)+S_{2}(t)\right)^{n}\right] & =\sum_{j=0}^{n}\left(\begin{array}{c}
n \\
j
\end{array}\right) S_{1}(0)^{j} S_{2}(0)^{n-j} \mathrm{E}\left[\exp \left(j X_{1}(t)+(n-j) X_{2}(t)\right)\right] \\
& =\sum_{j=0}^{n}\left(\begin{array}{c}
n \\
j
\end{array}\right) S_{1}(0)^{j} S_{2}(0)^{n-j} \exp \left(\psi_{\boldsymbol{X}}\left(-\mathrm{i}(j, n-j)^{\prime}\right) t\right),
\end{aligned}
$$

where we recall the cumulant function in (2.2). On the other hand, the $n$th moment of $S(t)$ is given by

$$
\mathrm{E}\left[S(t)^{n}\right]=S(0)^{n} \cdot \mathrm{E}\left[\mathrm{e}^{n X(t)}\right]=S(0)^{n} \exp \left(\psi_{X(t)}(-\mathrm{i} n)\right) .
$$

The cumulant function $\psi_{X(t)}$ includes the four univariate parameters $\alpha, \beta, \mu, \delta$, which for each $t>0$ can be found by moment matching, that is, by solving the four nonlinear equations

$$
S(0)^{n} \exp \left(\psi_{X(t)}(-\mathrm{i} n)\right)=\sum_{j=0}^{n}\left(\begin{array}{c}
n \\
j
\end{array}\right) S_{1}(0)^{j} S_{2}(0)^{n-j} \exp \left(\psi_{\boldsymbol{X}}\left(-\mathrm{i}(j, n-j)^{\prime} t\right)\right)
$$

for $n=1,2,3,4$. Note that we get one solution for each $t$, and the process $t \mapsto X(t)$ is not a Lévy process in general. We know that it is NIG distributed for each $t$, but the 
NIG parameters will change with time violating stationarity of the increments. If we insist on $X(t)$ being a NIG-Lévy process, we will have that $\psi_{X(t)}(s)=\psi_{X}(s) t$, where $\psi_{X}(s)$ is the cumulant function for $X(1)$. Hence, we can not any longer expect (3.4) to hold, but instead we can find parameters which minimize the distance in some norm between the expressions in (3.2) and (3.3). In applications like pricing of plain vanilla options, we are only interested in finding a NIG-distributed random variable $X(t)$ at a prescribed time $t$, being the exercise time of the option.

As we shall see in the numerical examples, there may be situations where the system of four nonlinear equations in (3.4) are hard to solve even numerically. We will therefore explore another approach which can be used to approximate a univariate NIG distribution. In a real life application, the bivariate $\log$ NIG assumption on the prices $S_{1}$ and $S_{2}$ means that the logreturn data are bivariate NIG distributed, and parameters can be estimated using for instance the method of moments or maximum likelihood. To approximate $S_{1}(t)+$ $S_{2}(t)$, the approach above tells us to match the moments implied by this bivariate model with the theoretical moments of $S(0) \exp (X(t))$, as in (3.4). An alternative to this method is to directly estimate $S(t)$ to the bivariate data, thereby avoiding the estimation of the bivariate NIG which by itself may be a challenging task in practice.

To discuss this approach further, define the logreturns of the process $S_{1}(t)+S_{2}(t)$ at time $i$ by

$$
Z(i)=\ln \left(\frac{S_{1}(i)+S_{2}(i)}{S_{1}(i-1)+S_{2}(i-1)}\right)
$$

for discrete time points $i=1, \ldots$. We assume that $Z(i)$ is independent and identically distributed, which means that $Z(i)$ comes from a univariate Lévy process. Denoting the expectation, variance, skewness and kurtosis of $Z$ by $m, v, s$ and $k$, respectively, and assuming $Z \sim \operatorname{NIG}(\alpha, \beta, \mu, \delta)$, we find after rearranging (2.9)-(2.12) expressions for $\alpha, \beta, \mu$ and $\delta$;

$$
\begin{aligned}
\alpha & =\sqrt{\frac{3 k-4 s^{2}-9}{v\left(k-\frac{5}{3} s^{2}-3\right)^{2}}} \\
\beta & =\frac{s}{\sqrt{v}\left(k-\frac{5}{3} s^{2}-3\right)} \\
\mu & =m-\frac{3 s \sqrt{v}}{3 k-4 s^{2}-9} \\
\delta & =3^{3 / 2} \frac{\sqrt{v\left(k-\frac{5}{3} s^{2}-3\right)}}{3 k-4 s^{2}-9} .
\end{aligned}
$$

Hence, by estimating the empirical mean, variance, skewness and kurtosis from observations of $Z(i)$, we can find the parameters of its NIG distribution. This could alternatively be 
done using maximum likelihood, of course. Thus, at time $t$, we have

$$
S_{1}(t)+S_{2}(t)=\left(S_{1}(0)+S_{2}(0)\right) \exp \left(\sum_{i=1}^{t} Z(i)\right),
$$

where we know from the independence of $Z(i)$ and the convolution property of the NIG that $S_{1}(t)+S_{2}(t) / S_{1}(0)+S_{2}(0)$ becomes $\operatorname{logNIG}$ distributed, with parameters $\alpha, \beta t, \mu t$ and $\delta$. In order to make the univariate dynamics risk-neutral, we perform an Esscher transform which entails in finding a parameter $\theta$ solving (2.5) in the one-dimensional case $(d=1)$. In practice this implies a modification of the skewness analogous to the bivariate case discussed above (see Benth, Groth and Kettler [6] for more details). In the simulation studies below we match using the terminal distribution at time $t$ instead of the logreturns. Moment matching on log scale is used, which means that we do not necessarily have a match of moments on price scale, as in (3.4). Note in passing that in many concrete situations, one models a basket of asset price series directly, for example, when modeling a stock index.

3.1. Numerical examples. The starting point of our numerical examples is the bivariate NIG parameters estimated by Rydberg [19] for two German data sets (1562 pairs of observations of Deutsche Bank and Dresdner Bank), the parameters being $\alpha=146.032$, $\boldsymbol{\beta}=(-31.284,27.515)^{\prime}, \boldsymbol{\mu}=(0.001,0.000)^{\prime}, \delta=0.011$, and

$$
\Delta=\left[\begin{array}{ll}
1.992 & 1.580 \\
1.580 & 1.756
\end{array}\right]
$$

The purpose of the numerical examples is to test the approximation of $S_{1}(t)+S_{2}(t)$ using a logNIG random variable. As observed in Henriksen [13] for the bivariate geometric Brownian motion case, the goodness of the lognormal approximation depends on the correlation between the two asset price processes. Thus, we set up different scenarios where we change the dependency structure between $X_{1}$ and $X_{2}$ in order to check the robustness of the approximation under different dependency structures. Further, we also check the robustness for extreme tails and/or skewness. Hence, we consider variations of the parameters estimated by Rydberg [19].

In all our examples, we let set $t=252^{1}$ and match the moments of $S_{1}(t)+S_{2}(t)$ with those of a $\log$ NIG variable using (3.4). Further, since we are going to price basket options in Section 4, we consider the risk-neutral dynamics of $S_{1}(t)$ and $S_{2}(t)$, that is, we solve the equations (2.7) and (2.8) for $\boldsymbol{\theta}=\left(\theta_{1}, \theta_{2}\right)$ such that $S_{1}$ and $S_{2}$ becomes $Q$-martingales. Recall from Section 2 that under $Q$, the only change in the bivariate NIG distribution for $\boldsymbol{X}(t)$ is in the skewness parameter, which becomes $\boldsymbol{\beta}_{Q}=\boldsymbol{\beta}+\boldsymbol{\theta}$. The risk-free interest rate is set equal to $4 \%$ annually, that is, $r=0.04 / 252$ on a daily time scale. For simplicity, we also assume $S_{1}(0)=S_{2}(0)=1$.

We consider 9 different scenarios, in which we consider various choices of $\boldsymbol{\Delta}$. The 9 scenarios are separated along two axes, one with various levels of correlation between $X_{1}$

\footnotetext{
${ }^{1}$ This corresponds to one financial year
} 


\begin{tabular}{l|l|l} 
Scenario 1: & Scenario 2: & Scenario $3:$ \\
Negative corr. & No corr. & Positive corr. \\
$X_{1}$ very low vol. & $X_{1}$ very low vol. & $X_{1}$ very low vol. \\
$X_{2}$ very high vol. & $X_{2}$ very high vol. & $X_{2}$ very high vol. \\
\hline Scenario 4: & Scenario 5: & Scenario 6: \\
Negative corr. & No corr. & Positive corr. \\
$X_{1}$ low vol. & $X_{1}$ low vol. & $X_{1}$ low vol. \\
$X_{2}$ high vol. & $X_{2}$ high vol. & $X_{2}$ high vol. \\
\hline Scenario 7: & Scenario 8: & Scenario 9: \\
Negative corr. & No corr. & Positive corr. \\
$X_{1}$ normal vol. & $X_{1}$ normal vol. & $X_{1}$ normal vol. \\
$X_{2}$ normal vol. & $X_{2}$ normal vol. & $X_{2}$ normal vol.
\end{tabular}

TABLE 1. An overview of the nine different scenarios split into different volatilities and correlations

and $X_{2}$, and the another with different levels of volatility. An overview of the nine scenarios are listed in Table 1.

The different choices of $\boldsymbol{\Delta}$ along with the implied correlations, $\boldsymbol{\beta}_{Q}$ 's and volatilities are listed in Table 2. The selection of $\boldsymbol{\Delta}$ was based on tuning the entries to reach desired levels of correlation while at the same time preserving reasonable levels of the parameters relative to the estimates in Rydberg [19].

The resulting estimates for $\alpha, \beta, \mu$ and $\delta$ from matching the four first moments in (3.4) are listed in Table 3 for the 9 scenarios. In Figure 1 we have plotted the moment matched $\log$ NIG distribution together with the distribution of $S_{1}(t)+S_{2}(t)$. The latter is based on simulation, using $10^{6}$ samples, and we have plotted the frequency axis on a log-scale to highlight potential differences in the tails. As we observe, the correspondence between the approximated distribution and the true is very good. The only deviation worth noting is a slight mismatch in the tails of the negatively correlated scenarios.

It is of interest to compare with the alternative approximation method, where we match $\ln \left(S_{1}(t)+S_{2}(t)\right)$ with a NIG variable. As we have discussed, it may in practice be easier to fit a univariate NIG variable directly to the observed logreturns of $S_{1}(t)+S_{2}(t)$. To check the results of this approach, we simulate $10^{6}$ outcomes of $S_{1}(t)+S_{2}(t)$ at time $t=252$ based on the bivariate $\log$ NIG distribution for $\left(S_{1}(t), S_{2}(t)\right)$ in the 9 different scenarios. Note that we do not simulate sample paths and calculate logreturns, but simulate the terminal distribution directly. This is more convenient, and equivalent as we have defined the method. However, in practice we only have available two time series of data, from which we would calculate the logreturns of the sum of the two series. The resulting estimated NIG parameters are listed in Table 4. Comparing with the figures in Table 3, we see that except for Scenario 1, the estimates are basically the same. The resulting probability densities for the 9 scenarios based on the simulations of $S_{1}(t)+S_{2}(t)$ are displayed in Figure 2, 

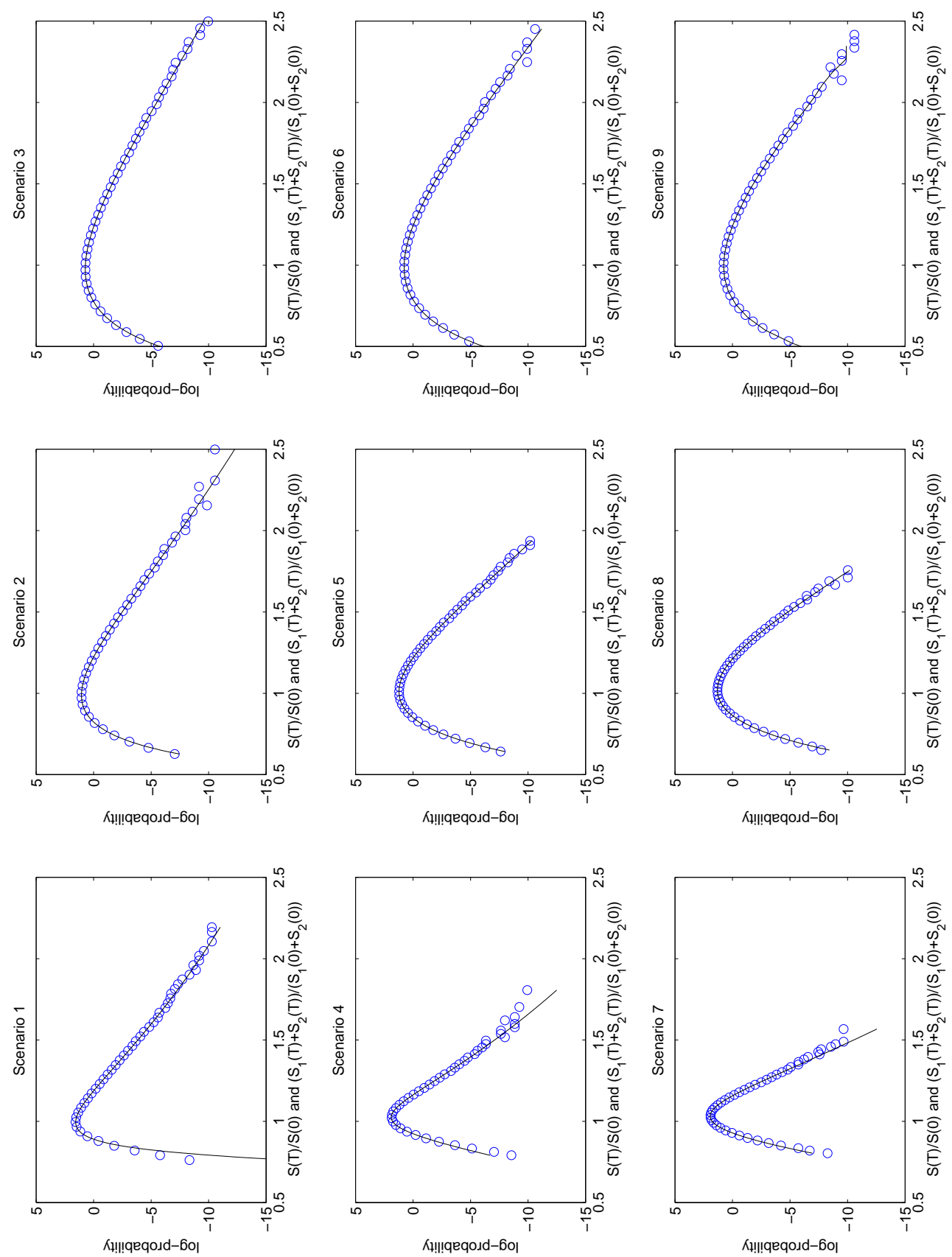

Figure 1. The logarithm of the density of $\left(S_{1}(t)+S_{2}(t)\right) /\left(S_{1}(0)+S_{2}(0)\right)$ as circles and $S(t) / S(0)$ as the whole line for the 9 scenarios based on moment matching through (3.4). 


\begin{tabular}{|c|c|c|c|c|c|}
\hline Scn. & \multicolumn{2}{|c|}{$\Delta$} & $\operatorname{Corr}\left(X_{1}, X_{2}\right)$ & $\boldsymbol{\beta}_{\mathrm{Q}}$ & Vol. $X_{1}$, Vol. $X_{2}$ \\
\hline 1 & {$\left[\begin{array}{r}0.87 \\
-1.5\end{array}\right.$} & $\begin{array}{c}-1.58 \\
4.00\end{array}$ & -0.84 & $(-45.75,-18.05)^{\prime}$ & $(0.13,0.28)^{\prime}$ \\
\hline 2 & {$\left[\begin{array}{c}0.2 \\
0\end{array}\right.$} & $\left.\begin{array}{c}0 \\
4.00\end{array}\right]$ & 0.00 & $(-44.66,0)^{\prime}$ & $(0.07,0.28)^{\prime}$ \\
\hline 3 & $\begin{array}{l}0.8 \\
1.5\end{array}$ & $\begin{array}{l}1.58 \\
4.00\end{array}$ & 0.84 & $(-46.00,18.19)^{\prime}$ & $(0.13,0.28)^{\prime}$ \\
\hline 4 & {$\left[\begin{array}{r}1.40 \\
-1.5\end{array}\right.$} & $\left.\begin{array}{c}-1.58 \\
2.50\end{array}\right]$ & -0.84 & $(-28.14,-17.45)^{\prime}$ & $(0.16,0.22)^{\prime}$ \\
\hline 5 & {$\left[\begin{array}{c}0.4 \\
0\end{array}\right.$} & $\left.\begin{array}{c}0 \\
2.50\end{array}\right]$ & 0.00 & $(-28.22,0.3365)^{\prime}$ & $(0.09,0.22)^{\prime}$ \\
\hline 6 & {$\left[\begin{array}{l}1.4 \\
1.5\end{array}\right.$} & $\left.\begin{array}{l}1.58 \\
2.50\end{array}\right]$ & 0.84 & $(-30.75,19.77)^{\prime}$ & $(0.16,0.22)^{\prime}$ \\
\hline 7 & {$\left[\begin{array}{r}1.99 \\
-1.5\end{array}\right.$} & $\left.\begin{array}{c}-1.58 \\
1.76\end{array}\right]$ & -0.84 & $(-19.34,-16.71)^{\prime}$ & $(0.20,0.18)^{\prime}$ \\
\hline 8 & {$\left[\begin{array}{r}0.5 \\
0\end{array}\right.$} & $\left.\begin{array}{c}0 \\
1.76\end{array}\right]$ & 0.00 & $(-20.01,0.69)^{\prime}$ & $(0.10,0.18)^{\prime}$ \\
\hline 9 & $\begin{array}{l}1.9 \\
1.5 \\
\end{array}$ & $\left.\begin{array}{l}1.58 \\
1.76\end{array}\right]$ & 0.84 & $(-23.14,21.51)^{\prime}$ & $(0.20,0.18)^{\prime}$ \\
\hline
\end{tabular}

TABLE 2. Correlation and yearly volatility in the 9 scenarios based on the Rydberg parameters

\begin{tabular}{ccccc} 
Scn & $\alpha$ & $\beta$ & $\mu$ & $\delta$ \\
\hline 1 & 163.66 & 145.30 & -0.23 & 0.14 \\
2 & 209.7 & 179.3 & -0.94 & 0.59 \\
3 & 96.27 & 41.53 & -1.27 & 2.70 \\
4 & 37.66 & 11.19 & -0.008 & 0.14 \\
5 & 78.53 & 34.03 & -0.35 & 0.79 \\
6 & 127.86 & 10.23 & -0.32 & 4.28 \\
7 & 56.05 & 9.75 & 0.007 & 0.18 \\
8 & 92.90 & 21.16 & -0.19 & 0.95 \\
9 & 171.62 & -3.56 & 0.14 & 5.67 \\
\hline
\end{tabular}

TABLE 3. Univariate NIG parameters found from solving the nonlinear system of equations (3.4)

where the frequency axis is on a log-scale. There is not much discrepancy between the two densities in the 9 scenarios. We notice that also here there is a slight mismatch in the tails of the negatively correlated scenarios. To further validate the results from the simulation 

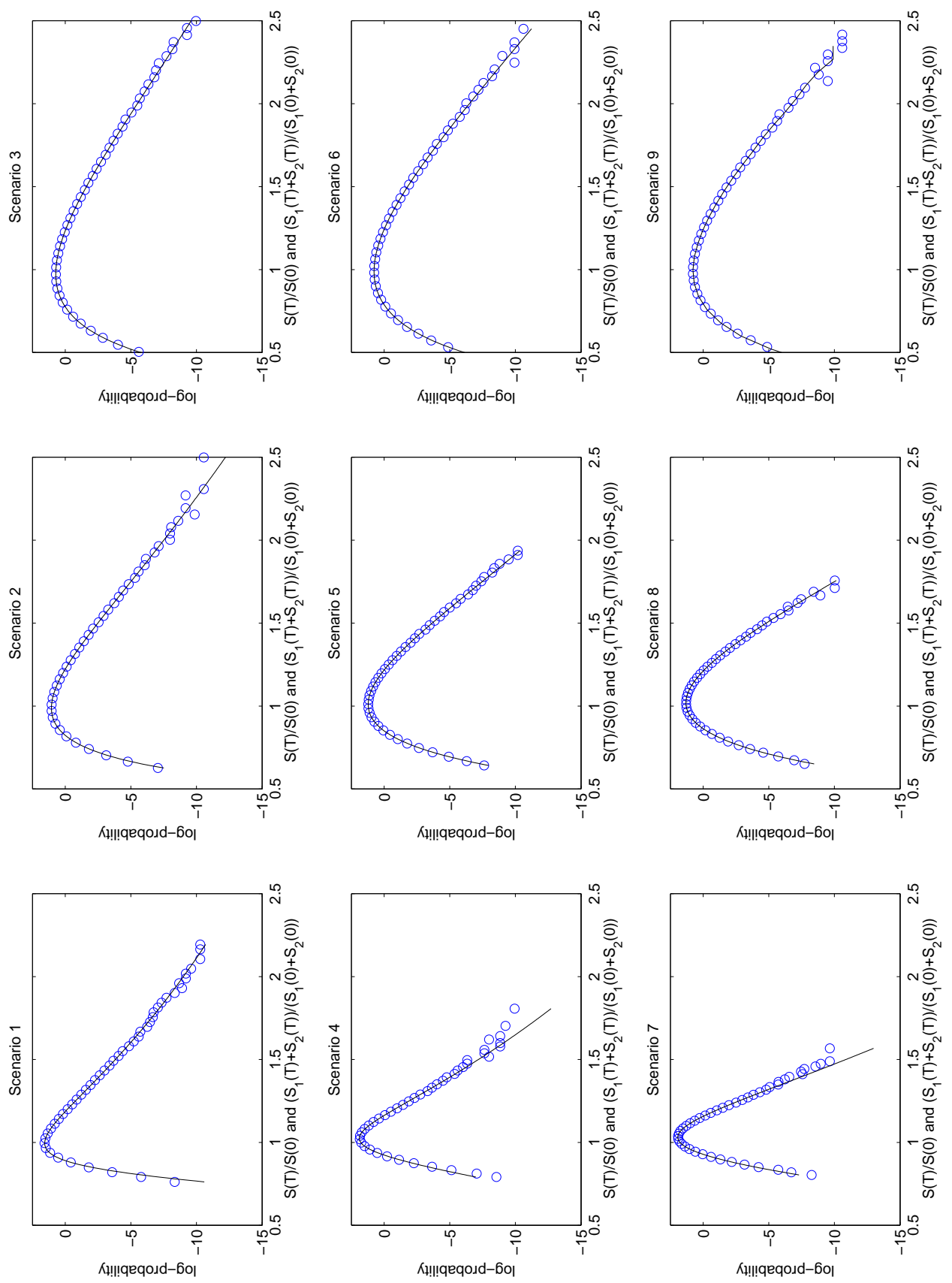

FiguRE 2. The log probability of $\left(S_{1}(t)+S_{2}(t)\right) /\left(S_{1}(0)+S_{2}(0)\right)$ as circles and $S(t) / S(0)$ as the whole line for the 9 scenarios based on simulation of $S_{1}(t)+S_{2}(t)$ and moment matching through (3.6)-(3.9) 


\begin{tabular}{ccccc} 
Scn & $\alpha$ & $\beta$ & $\mu$ & $\delta$ \\
\hline 1 & 70.79 & 53.90 & -0.16 & 0.17 \\
2 & 209.6 & 179.5 & -0.93 & 0.58 \\
3 & 97.08 & 41.87 & -1.28 & 2.73 \\
4 & 39.94 & 12.56 & -0.008 & 0.14 \\
5 & 76.95 & 32.60 & -0.34 & 0.79 \\
6 & 128.58 & 5.82 & -0.17 & 4.34 \\
7 & 61.96 & 12.05 & -0.000 & 0.19 \\
8 & 92.53 & 22.14 & -0.20 & 0.94 \\
9 & 171.59 & -4.79 & 0.18 & 5.66 \\
\hline
\end{tabular}

TABLE 4. Univariate NIG parameters found from moment matching the simulations (3.6)-(3.9)

\begin{tabular}{ccccccccc} 
Scn. & Mean & Mean & Var & Var & Skew & Skew & Kurt & Kurt \\
& $S_{1}+S_{2}$ & $S$ & $S_{1}+S_{2}$ & $S$ & $S_{1}+S_{2}$ & $S$ & $S_{1}+S_{2}$ & $S$ \\
\hline 1 & 2.0816 & 2.0816 & 0.0403 & 0.0402 & 1.2351 & 1.2276 & 6.0207 & 6.0571 \\
2 & 2.0819 & 2.0822 & 0.0921 & 0.0922 & 0.8002 & 0.8027 & 4.2353 & 4.2635 \\
3 & 2.0809 & 2.0814 & 0.1719 & 0.1715 & 0.7026 & 0.6999 & 3.9359 & 3.9364 \\
4 & 2.0816 & 2.0816 & 0.0180 & 0.0180 & 0.6785 & 0.6721 & 4.5283 & 4.4292 \\
5 & 2.0815 & 2.0816 & 0.0614 & 0.0615 & 0.5482 & 0.5460 & 3.6226 & 3.6137 \\
6 & 2.0823 & 2.0819 & 0.1494 & 0.1494 & 0.5717 & 0.5720 & 3.6023 & 3.5905 \\
7 & 2.0816 & 2.0816 & 0.0146 & 0.0145 & 0.3703 & 0.3644 & 3.5610 & 3.5257 \\
8 & 2.0814 & 2.0812 & 0.0489 & 0.0488 & 0.4062 & 0.4017 & 3.3438 & 3.3328 \\
9 & 2.0811 & 2.0804 & 0.1455 & 0.1451 & 0.5534 & 0.5534 & 3.5473 & 3.5442 \\
\hline
\end{tabular}

TABle 5. Mean, variance, skewness and kurtosis of $S_{1}+S_{2}$ and $S$ in the 9 scenarios.

based example, we report the statistics from the study in Table 5. The moments of $S(t)$ agree to a high degree with those of $S_{1}(t)+S_{2}(t)$. Recall that the moments of $\ln S(t)$ and $\ln \left(S_{1}(t)+S_{2}(t)\right)$ are equal since they have been matched. However, it is not immediate that the moments reported in Table 5 should resemble, since we exponentiate the random variables. A careful inspection of the results in Table 5 reveals that the skewness and kurtosis estimates seem to deviate slightly from the ones of $S_{1}(t)+S_{2}(t)$. Based on the statistics for the four first moments and the probability density plots, we conclude that the NIG approximation through (3.6)-(3.9) for the 9 considered scenarios perform excellently. Obviously, when matching the moments by solving (3.4), the statistics coincide perfectly.

To check the univariate approximations in more extreme situations, we changed the parameters $\alpha$ and $\delta$ to introduce more skewness and kurtosis in the bivariate NIG distribution. We assume $\alpha=60$ and $\delta=0.055$, whilst $\boldsymbol{\beta}$ and $\boldsymbol{\mu}$ remain unchanged. We consider the 9 different scenarios of $\boldsymbol{\Delta}$ as above, but now the resulting risk-neutral skewnesses $\boldsymbol{\beta}_{Q}$ 


\begin{tabular}{|c|c|c|}
\hline Scn & $\boldsymbol{\beta}_{\mathrm{Q}}$ & $\left(\operatorname{Vol} . X_{1}, \text { Vol. } X_{2}\right)^{\prime}$ \\
\hline 1 & $(-8.30,-3.73)^{\prime}$ & $(0.45,0.96)^{\prime}$ \\
\hline & $2(-4.17,-0.46)^{\prime}$ & $(0.24,0.96)^{\prime}$ \\
\hline & $3 \quad(-2.53,0.54)^{\prime}$ & $(0.45,0.96)^{\prime}$ \\
\hline & $4 \quad(-5.74,-4.06)^{\prime}$ & $(0.57,0.76)^{\prime}$ \\
\hline & $5 \quad(-2.79,-0.43)^{\prime}$ & $(0.30,0.76)^{\prime}$ \\
\hline & $6 \quad(-2.34,1.05)^{\prime}$ & $(0.57,0.76)^{\prime}$ \\
\hline & $7 \quad(-4.47,-4.42)^{\prime}$ & $(0.68,0.64)^{\prime}$ \\
\hline & $8(-2.11,-0.40)^{\prime}$ & $(0.36,0.64)^{\prime}$ \\
\hline & $9 \quad(-2.25,1.62)^{\prime}$ & $(0.68,0.64)^{\prime}$ \\
\hline
\end{tabular}

TABLE 6 . Correlation and volatility in the case of heavy skewness and kurtosis for the 9 scenarios

and volatilities change since we have different $\alpha$ and $\delta$ parameters. The correlations remain unchanged. We report the figures in Table 6.

It turns out that the moment matching technique based on solving (3.4) is more challenging in the extreme situation. There are several reasons for this; i) some of the parameters become very large - this causes numerical problems as the equations contain terms taking exponentials of these parameters, ii) the sensitivity of the parameters is significant - small changes in the parameters makes the solutions diverge, iii) there is no natural starting point for the nonlinear numerical solver which makes the process time consuming - in the normal situation we used the solutions from equations (3.6)-(3.9) as starting points, but in the extreme situation this generates imaginary numbers in some scenarios. From Table 7 it can be seen that we have only been able to derive exact solutions in scenario 6,8 and 9. The rest of the scenarios have approximative solutions where the right side of (3.4) deviates from the left side by a magnitude of $10^{-3}-10^{-2}$, where the first four scenarios gave the worst approximations. Worth noticing is the extreme values for $\delta$ in some of the scenarios. The resulting probability distributions based on the parameters from Table 7 are given in Figure 3. The results are surprisingly good taken into consideration that most of the parameters are approximate solutions of (3.4). One overall trend is that the tails of $S_{1}+S_{2}$ are heavier than the tails of the moment matched $S$.

Again, we wish to compare the method based on simulation of $\ln \left(S_{1}(t)+S_{2}(t)\right)$ and matching of the log-prices. In contrast to solving (3.4), this approach easily provides estimates in all the scenarios. In Table 8 we have listed the estimated NIG parameters using this method. Compared to Table 7 we see a significant difference in the parameters. Particularly in scenario 1, 2, 4 and 7 the difference in relationship between $\alpha$ and $\delta$, which controls the tail heaviness, is apparent. The resulting moments for the approximation compared to $S_{1}(t)+S_{2}(t)$ are reported in Table 9 . We clearly observe that the skewness and kurtosis severely fail to match in some of the scenarios, whereas the expectation and variance are relatively close. The mismatch increases with the order of the moments. 



Figure 3. The log probability of $\left(S_{1}(t)+S_{2}(t)\right) /\left(S_{1}(0)+S_{2}(0)\right)$ as circles and $S(t) / S(0)$ as the whole line for the 9 extreme scenarios based on simulation of $S_{1}(t)+S_{2}(t)$ and moment matching through (3.4). In scenario 9 the whole line is based on simulations because of numerical problems in the NIG probability density function. 


\begin{tabular}{ccccc} 
Scn & $\alpha$ & $\beta$ & $\mu$ & $\delta$ \\
\hline 1 & $\mathbf{2 4 . 7 5}$ & $\mathbf{1 9 . 8 9}$ & $\mathbf{- 1 . 8 4}$ & $\mathbf{1 . 2 6}$ \\
2 & $\mathbf{2 6 . 0 8}$ & $\mathbf{2 0 . 5 5}$ & $\mathbf{- 2 . 4 4}$ & $\mathbf{1 . 7 9}$ \\
3 & $\mathbf{1 9 . 0 6}$ & $\mathbf{1 0 . 9 5}$ & $\mathbf{- 3 . 4 6}$ & $\mathbf{4 . 6 5}$ \\
4 & $\mathbf{2 0 1 . 6 4}$ & $\mathbf{1 9 6 . 4 9}$ & $\mathbf{- 1 . 0 0}$ & $\mathbf{0 . 2 3}$ \\
5 & $\mathbf{1 0 . 6 6}$ & $\mathbf{4 . 7 3}$ & $\mathbf{- 0 . 7 0}$ & $\mathbf{1 . 3 1}$ \\
6 & 25.93 & 5.59 & -2.30 & 9.68 \\
7 & $\mathbf{1 1 . 8 5}$ & $\mathbf{6 . 4 1}$ & $\mathbf{- 0 . 4 4}$ & $\mathbf{0 . 6 6}$ \\
8 & 11.99 & 3.91 & -0.49 & 1.35 \\
9 & 42.69 & 0.16 & -0.23 & 17.05 \\
\hline
\end{tabular}

TABLE 7. Univariate NIG parameters found from solving the nonlinear system of equations (3.4) in the heavy skewness and kurtosis case. The parameters in bold are approximative solutions to the nonlinear equations. These were the best solutions we could find numerically, and the errors (right hand side less left hand side of (3.4)) are in the order of $10^{-3}-10^{-2}$.

\begin{tabular}{ccccc} 
Scn & $\alpha$ & $\beta$ & $\mu$ & $\delta$ \\
\hline 1 & 22.55 & 20.04 & -0.529 & 0.26 \\
2 & 24.41 & 21.16 & -1.11 & 0.59 \\
3 & 18.23 & 11.63 & -3.02 & 3.43 \\
4 & 10.22 & 6.55 & -0.34 & 0.40 \\
5 & 10.08 & 5.08 & -0.63 & 1.00 \\
6 & 23.34 & 4.68 & -1.97 & 8.82 \\
7 & 11.25 & 6.96 & -0.35 & 0.44 \\
8 & 11.59 & 3.43 & -0.45 & 1.35 \\
9 & 36.52 & -0.09 & -0.12 & 14.55 \\
\hline
\end{tabular}

TABLE 8. Univariate NIG parameters found from moment matching the simulated log-prices using (3.6)-(3.9) in the heavy skewness and kurtosis case

Particularly the scenarios 1, 2, 4 and 7 give bad results, in line with the failure of the moment matching technique.

We have visualized the difference by plotting the logarithm of the two distributions resulting from the simulation based approximation for the 9 scenarios in Figure 4 . It is evident that the probability densities of $S(t)$ in scenario 1, 2, 4 and 7 differ from those of $S_{1}(t)+S_{2}(t)$. These are also the cases where the moments differ the most. We notice that except for scenario 2, all these have negative correlation. In Zacks and Tsokos [22] the moment matching approximation in the log normal case turns out to be very good for positive (including zero) correlations, while for negative correlations it works poorly. 

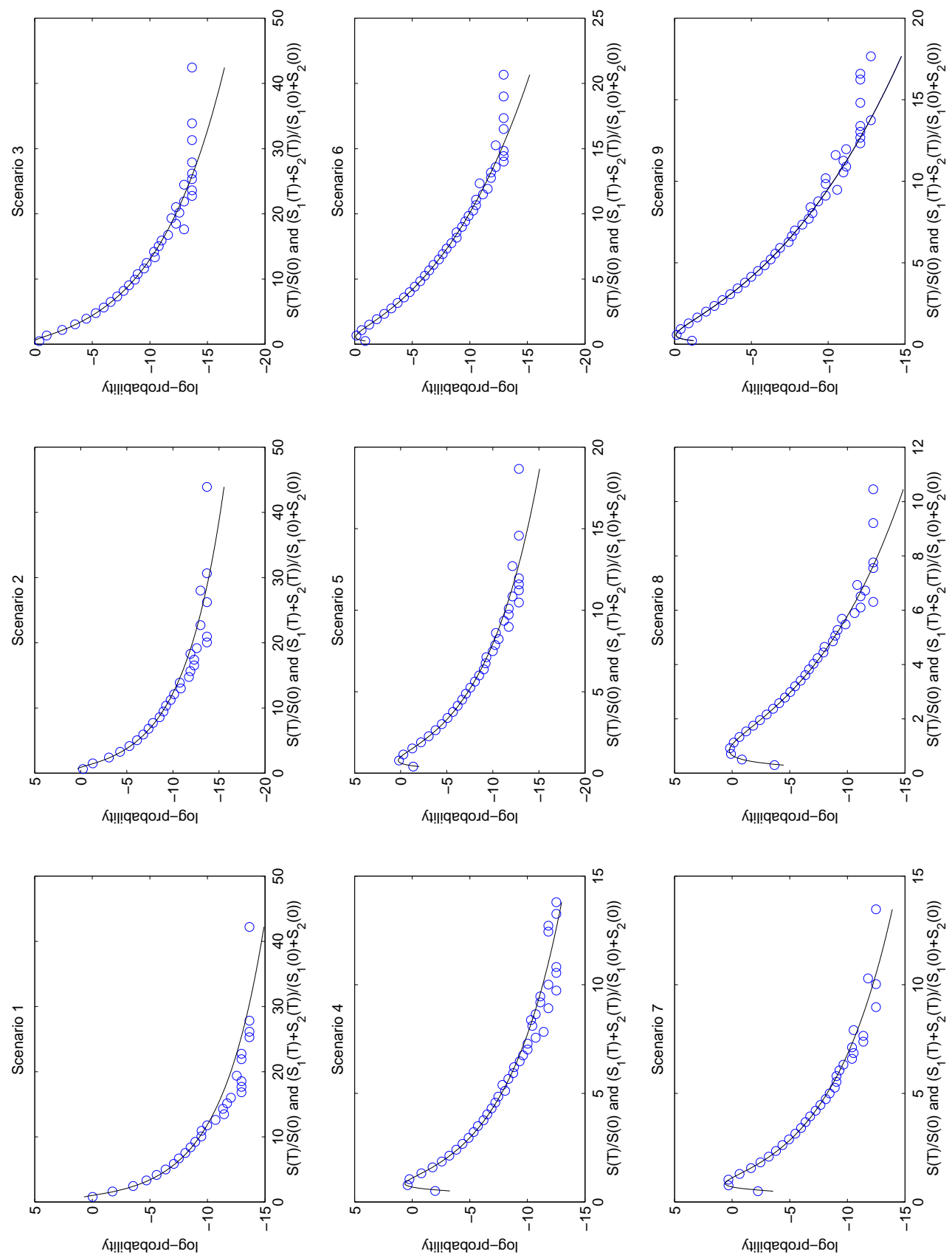

FiguRE 4 . The log probability of $\left(S_{1}(t)+S_{2}(t)\right) /\left(S_{1}(0)+S_{2}(0)\right)$ as circles and $S(t) / S(0)$ as the whole line for the 9 extreme scenarios based on simulation of $S_{1}(t)+S_{2}(t)$ and moment matching through (3.6)-(3.9). 


\begin{tabular}{ccccccccc} 
Scn & Mean & Mean & Var & Var & Skew & Skew & Kurt & Kurt \\
& $S_{1}+S_{2}$ & $S$ & $S_{1}+S_{2}$ & $S$ & $S_{1}+S_{2}$ & $S$ & $S_{1}+S_{2}$ & $S$ \\
\hline 1 & 2.0797 & 2.0821 & 1.2233 & 1.4222 & 6.9206 & 18.322 & 129.319 & 1394.50 \\
2 & 2.0823 & 2.0822 & 1.7183 & 1.8215 & 5.2701 & 7.9346 & 82.3564 & 236.693 \\
3 & 2.0809 & 2.0768 & 2.8353 & 2.8348 & 4.0823 & 4.5538 & 47.3974 & 77.2334 \\
4 & 2.0825 & 2.0843 & 0.6060 & 0.6223 & 3.7676 & 4.7936 & 36.5736 & 77.1429 \\
5 & 2.0813 & 2.0812 & 0.9482 & 0.9455 & 2.7967 & 2.9244 & 22.1769 & 28.4213 \\
6 & 2.0821 & 2.0847 & 2.2226 & 2.2314 & 2.6362 & 2.7134 & 17.5144 & 19.9847 \\
7 & 2.0809 & 2.0810 & 0.5158 & 0.5186 & 3.0600 & 3.3986 & 24.7385 & 39.6986 \\
8 & 2.0826 & 2.0816 & 0.6936 & 0.6923 & 1.7967 & 1.8079 & 10.2423 & 10.8867 \\
9 & 2.0814 & 2.0798 & 2.1261 & 2.1265 & 2.4849 & 2.4560 & 16.0137 & 15.1263 \\
\hline
\end{tabular}

TABLE 9. Mean, variance, skewness and kurtosis of $S_{1}+S_{2}$ and $S$ in the case of $\alpha=60$ and $\delta=0.055$.

Compared to Figure 4, we see that the simulation based approximation gives heavier tails and more kurtosis. This seems to give a less good overall fit, but the approximation of the true distribution is still quite good.

From the above examples we conclude that the moment matching approach using (3.4) provides the best approximation. However, it may be less time efficient and more technically challenging as a set of nonlinear equations has to be solved numerically. The alternative approach based on log-price matching seems to be more robust when it comes to estimating the parameters and significantly more time efficient. On the other hand, it seems that the log-based approximation is less accurate in the extreme case, whereas the two methods give very similar results for "reasonable" choices of parameters. Negative correlation between $S_{1}$ and $S_{2}$ result in less good approximations for both methods.

To shed some light on why the negative correlation case does not seem to be as good as the positive, let us do the following Taylor expansion,

$$
\begin{aligned}
2 \ln \left(\mathrm{e}^{X_{1}(t)}+\mathrm{e}^{X_{2}(t)}\right)=X_{1}(t)+X_{2}(t)+\ln \left(1+\mathrm{e}^{X_{2}(t)-X_{1}(t)}\right) & \\
& +\ln \left(1+\mathrm{e}^{X_{1}(t)-X_{2}(t)}\right) \\
=X_{1}(t)+X_{2}(t)+\frac{1}{2}\left(\cosh \left(X_{1}(t)-X_{2}(t)\right)-1\right) & +\ln (2)+O\left(\left(X_{1}(t)-X_{2}(t)\right)^{4}\right) .
\end{aligned}
$$

If $X_{1}(t) \sim \operatorname{NIG}\left(\alpha, \beta, \mu_{1}, \delta_{1}\right)$ and $X_{2}(t) \sim \operatorname{NIG}\left(\alpha, \beta, \mu_{2}, \delta_{2}\right)$ are independent, then $X_{1}(t)+$ $X_{2}(t) \sim \operatorname{NIG}\left(\alpha, \beta, \mu_{1}+\mu_{2}, \delta_{1}+\delta_{2}\right)$. Thus, provided that the other terms on the right hand side of (3.10) are small, we have that the left hand side is close to being NIG distributed. If $X_{1}$ and $X_{2}$ are dependent, we see that this can scale down or up the remainder terms. In particular, $X_{1}-X_{2}$ can become big if we have a negative correlation, while for positively correlated variables this difference will be smaller since the two random variables tend to co-move. Similar considerations hold for the cosh-term. Even though $X_{1}$ and $X_{2}$ may not be NIG when the two are dependent, they will be reasonably close to such a distribution. In 
the negative correlation case we then see that the remainder terms move the distribution of $\ln \left(\exp \left(X_{1}(t)\right)+\exp \left(X_{2}(t)\right)\right)$ away from being NIG. This informal discussion gives us some indications on why the negative correlation case works badly in the NIG case (as in the lognormal case). In fact, it points towards that all distributions which are closed under convolution can only be poorly approximated by the methods we have considered when the correlation is negative.

\section{PRicing BASket options USing NIG approximations}

In this section we will price European call basket options based on two assets to study the relationship between the moment matching approximation errors and the option prices. We wish to analyse if there is a smoothing mechanism in the pricing valuation that makes option prices similar even in the cases when the NIG approximation does not provide a good fit. The approximation $S(t)$ of $S_{1}(t)+S_{2}(t)$ in this Section is based on moment matching of the log-prices.

The price of a European call basket option with strike $K$ and exercise time $t$ is

$$
p_{S_{1}+S_{2}}=\mathrm{e}^{-r t} \mathrm{E}_{Q}\left[\left(S_{1}(t)+S_{2}(t)-K\right)^{+}\right] .
$$

The expectation is difficult to calculate analytically in the bivariate $\log$ NIG case, and simulation procedures must be used. We approximate the price $p_{S_{1}+S_{2}}$ by

$$
p_{S}=\mathrm{e}^{-r t} \mathrm{E}_{Q}\left[(S(t)-K)^{+}\right]
$$

where $S(t)$ is the univariate $\log$ NIG variable approximating $S_{1}(t)+S_{2}(t)$. Note that $S(t)$ is approximating the risk-neutral version of $S_{1}(t)+S_{2}(t)$, and therefore the $Q$-probability in (4.2) refers to the same as in (4.1), and is not necessarily a measure making $S(t)$ risk-neutral. The price $p_{S}$ can be efficiently calculated using transform-based methods, like for instance fast Fourier Transform (FFT). We refer to Carr and Madan [9] for a detailed exposition of the use of FFT in option pricing where the cumulant function of the random variable involved is known. Since our concern is to evaluate the goodness of the approximation (4.2) of (4.1), we use Monte Carlo simulations to calculate both prices $p_{S_{1}+S_{2}}$ and $p_{S}$. Recall that we use a yearly interest rate of $4 \%$ and $S_{1}(0)=S_{2}(0)=1$, and in all the examples we choose the exercise time to be $t=252$.

Based on $10^{6}$ simulated outcomes of $S_{1}(t)+S_{2}(t)$ and $S(t)$, we calculate $p_{S_{1}+S_{2}}$ and $p_{S}$ for the strikes $K=1,2,3$ and 4 . The results are presented in Table 10 for the 9 normal scenarios. We hardly observe any difference between the "true" price $p_{S_{1}+S_{2}}$ and approximation $p_{S}$. This is as expected, since the distributions of $S_{1}(t)+S_{2}(t)$ and $S(t)$ were seen to be very close for these scenarios. There are minor differences, mostly below $0.5 \%$ deviation from $p_{S_{1}+S_{2}}$, except in scenario 3 for the strike $K=3$, where the difference is around $1.5 \%$. We checked for Monte Carlo error in the prices using the FFT method for $p_{S}$, which showed that the errors had a neglible magnitude.

We move on to the 9 extreme scenarios. Recall that even though the four first moments of $\ln S(t)$ and $\ln \left(S_{1}(t)+S_{2}(t)\right)$ match (by definition of the matching process), we observed severe deviations in the distributions in scenarios 1,2, 4 and 7 . The simulated prices are reported in Table 11 . The approximated prices are basically equal to the true simulated 


\begin{tabular}{ccccccccc} 
& \multicolumn{2}{c}{$K=1$} & \multicolumn{2}{c}{$K=2$} & \multicolumn{2}{c}{$K=3$} & \multicolumn{2}{c}{$K=4$} \\
\hline Scn & $S_{1}+S_{2}$ & $S$ & $S_{1}+S_{2}$ & $S$ & $S_{1}+S_{2}$ & $S$ & $S_{1}+S_{2}$ & $S$ \\
\hline 1 & 1.0392 & 1.0392 & 0.1137 & 0.1139 & 0.0003 & 0.0003 & 0.0000 & 0.0000 \\
2 & 1.0394 & 1.0398 & 0.1536 & 0.1539 & 0.0016 & 0.0016 & 0.0000 & 0.0000 \\
3 & 1.0386 & 1.0390 & 0.1949 & 0.1950 & 0.0067 & 0.0066 & 0.0001 & 0.0001 \\
4 & 1.0392 & 1.0394 & 0.0966 & 0.0965 & 0.0000 & 0.0000 & 0.0000 & 0.0000 \\
5 & 1.0391 & 1.0392 & 0.1356 & 0.1357 & 0.0002 & 0.0002 & 0.0000 & 0.0000 \\
6 & 1.0399 & 1.0395 & 0.1867 & 0.1864 & 0.0040 & 0.0040 & 0.0000 & 0.0000 \\
7 & 1.0392 & 1.0392 & 0.0941 & 0.0939 & 0.0000 & 0.0000 & 0.0000 & 0.0000 \\
8 & 1.0390 & 1.0388 & 0.1271 & 0.1269 & 0.0000 & 0.0000 & 0.0000 & 0.0000 \\
9 & 1.0387 & 1.0381 & 0.1843 & 0.1837 & 0.0035 & 0.0035 & 0.0000 & 0.0000 \\
\hline
\end{tabular}

TABLE 10. Basket option prices with four decimals of accuracy for different strikes in the 9 normal scenarios

\begin{tabular}{ccccccccc} 
& \multicolumn{2}{c}{$K=1$} & \multicolumn{2}{c}{$K=2$} & \multicolumn{2}{c}{$K=3$} & \multicolumn{2}{c}{$K=4$} \\
\hline Scn & $S_{1}+S_{2}$ & $S$ & $S_{1}+S_{2}$ & $S$ & $S_{1}+S_{2}$ & $S$ & $S_{1}+S_{2}$ & $S$ \\
\hline 1 & 1.0374 & 1.0397 & 0.3158 & 0.3250 & 0.1385 & 0.1366 & 0.0781 & 0.0749 \\
2 & 1.0441 & 1.0432 & 0.4100 & 0.4118 & 0.1945 & 0.1914 & 0.1079 & 0.1051 \\
3 & 1.0909 & 1.0872 & 0.5535 & 0.5500 & 0.3022 & 0.2991 & 0.1781 & 0.1758 \\
4 & 1.0401 & 1.0418 & 0.2713 & 0.2752 & 0.0775 & 0.0770 & 0.0309 & 0.0305 \\
5 & 1.0424 & 1.0422 & 0.3542 & 0.3547 & 0.1215 & 0.1202 & 0.0493 & 0.0480 \\
6 & 1.0914 & 1.0936 & 0.5309 & 0.5322 & 0.2652 & 0.2658 & 0.1397 & 0.1401 \\
7 & 1.0386 & 1.0386 & 0.2629 & 0.2652 & 0.0665 & 0.0649 & 0.0231 & 0.0221 \\
8 & 1.0433 & 1.0423 & 0.3284 & 0.3279 & 0.0859 & 0.0860 & 0.0252 & 0.0248 \\
9 & 1.0904 & 1.0891 & 0.5258 & 0.5253 & 0.2578 & 0.2575 & 0.1321 & 0.1321 \\
\hline
\end{tabular}

TABLE 11. Basket option prices with four decimals of accuracy for different strikes in the 9 scenarios with heavy skewness and kurtosis

prices for $K=1$, taking the possible Monte Carlo errors into account. This is the case for all scenarios, indicating that there is a clear smoothing effect for options in-the-money. For at-the-money options, there is a slight tendency towards an increasing error, although the prices also in this case look very similar. For scenarios 1 and 4 the errors are $2.9 \%$ and $1.4 \%$ respectively, overestimating the price. Scenario 7 gives an overestimation of $0.87 \%$, while scenario 2 only $0.43 \%$. It is interesting to note that all the "bad" cases give an overestimation of the price, which is not true for $K=1$. The remaining errors for $K=2$ are in the range of $0.5 \%$ in absolute value. Going to out-of-the-money options, the approximated prices start to underestimate the true value. Basically all the approximated prices are below the "true" ones, a clear sign of the difference in tail heaviness between $S(t)$ and $S_{1}(t)+S_{2}(t)$. The option prices indicate that $S(t)$ must have lighter tails than 
$S_{1}(t)+S_{2}(t)$, which is in line with what we observed when comparing the distributions. The errors are generally increasing when moving from $K=3$ to $K=4$, also in line with the differences in distributional tails between the approximated and the true random variables. Scenarios 1 and 7 have errors $-1.4 \%$ and $-2.4 \%$ for $K=3$, and $-4.1 \%$ and $-4.3 \%$ for $K=4$, respectively. The errors for scenarios 2 and 4 are lower, but still among the worst of all the scenarios. However, we see that overall there is a smoothing effect, and the mismatch in distributions do not imply necessarily a huge pricing error, even for far out-of-the-money options.

\section{Conclusions And Future WORK}

In this paper we have analysed numerically the approximation of a sum of two dependent $\log$ NIG variables with a univariate $\log$ NIG variable. The motivation for the study is the fast evaluation of basket options, where the marginal log-prices are modeled as dependent normal inverse Gaussian Lévy processes. We have analysed the approximation in terms of distributional fit and pricing of basket call options. The overall conclusion is that a sum of $\log$ NIG variables can be reasonably well approximated by a univariate logNIG variable. However, in particular when there is a negative correlation between the two NIG variables, the approximation may fail, producing a big mismatch in the tails.

We have based the approximation on the matching of moments. However, in some extreme cases, this approach is numerically challenging and time consuming. It is demonstrated the the matching of log variables is more efficient, however, this approach does not necessarily give as good a fit. On the other hand, it seems that the mismatch is in the right tails, and experiments with call option pricing show a robust pricing approximation. The error is increasing when moving towards far out-of-the-money options, but the levels of mispricing is not very high.

Our results indicate that the approximation of a sum of $\log$ NIG variables by a univariate $\log$ NIG works well. However, for cases with negative correlation, one must be careful when applying the approximation to the pricing of far out-of-the-money call options. More research is needed in order to analyse higher dimensional problems, and different types of basket options. Such investigations may also involve using other approximations than the univariate $\log$ NIG.

\section{REFERENCES}

[1] Abramowitz, M. and Stegun, I. A. (1972). Handbook of Mathematical Functions. Dover: New York

[2] Asmussen, S., and Rojas-Nandayapa, L. (2006). Sums of dependent lognormal random variables: asymptotics and simulation. Research Report, Thiele Centre, University of Aarhus

[3] Barndorff-Nielsen, O. E. (1977). Exponentially decreasing distributions for the logarithm of particle size. Proc. R. Stat. Soc., A 353, pp. 401-419.

[4] Barndorff-Nielsen, O. E. (1978). Hyperbolic distributions and distributions on hyperbolae. Scand. J. Statist., 5, pp 151-157.

[5] Barndorff-Nielsen, O. E. (1997). Normal inverse Gaussian distributions and stochastic volatility modeling. Scand. J. Statist., 24, pp. 1-13. 
[6] Benth, F. E., Groth, M., and Kettler, P. C. (2006). A quasi-Monte Carlo algorithm for the normal inverse Gaussian distribution and valuation of financial derivatives. Intern. J. Theoret. Applied Finance, 9, pp 843-868

[7] Blæsild, P. (1981). The two-dimensional hyperbolic distribution and related distributions, with an application to Johannsen's bean data. Biometrika, 68(1), pp. 251-263.

[8] Börger, R. (2007). Energy-Related Commodity Futures. PhD thesis, Universität Ulm.

[9] Carr, P., and Madan, D. (1999). Option valuation using the fast Fourier transform. J. Comp. Finance, 2, pp. 61-73.

[10] Dagpunar, J. S. (1989). An easily implemented generalized inverse Gaussian generator. Commun. Statist. Simul., 18(2), pp. 703-710.

[11] Eberlein, E. and Keller, U. (1995). Hyperbolic distributions in finance. Bernoulli, 1, pp. 281-299.

[12] Fama, E. F. (1970). Efficient markets: a review of theory and empirical work. J. Finance, May, pp. 383-417.

[13] Henriksen, P. N. (2008). Lognormal moment matching and pricing of basket options. E-print, University of Oslo.

[14] Hoedemakers, T. (2005). Modern Reserving Techniques for the Insurance Business. PhD Thesis, Katholieke Universiteit Leuven.

[15] Levy, E. (1992). Pricing European average rate currency options. J. Intern. Money Finance, 11(5), pp. $474-491$.

[16] Lillestøl, J. (2002). Some crude approximation, calibration and estimation procedures for NIGvariates. Working paper, The Norwegian School of Economics and Business Administration.

[17] Lillestøl, J. (2006). Some new bivariate IG and NIG-distributions for modeling covariate financial returns. Working paper, The Norwegian School of Economics and Business Administration.

[18] Prause, K. (1999). The Generalized Hyperbolic Model: Estimation, Financial Derivatives and Risk Measures. PhD Thesis, Albert-Ludwigs-Universität Freiburg.

[19] Rydberg, T. H. (1997). The normal inverse Gaussian Levy process: simulation and approximation. Commun. Statist. Stoch. Models, 13, pp. 887-910.

[20] Shiryaev, A. N. (1999). Essentials of Stochastic Finance. World Scientific.

[21] Turnbull, S. M. and Wakeman, L. M. (1991). A Quick Algorithm for Pricing European Average Options. J. Financial Quantit. Anal., 26, pp. 377-389.

[22] Zacks, S. and Tsokos, C. P. (1978). The distribution of sums of dependent log-normal variables. Technical Report No 31, Defense Technical Information Center.

(Fred Espen Benth), Centre of Mathematics for Applications, University of Oslo, P.O. Box 1053, Blindern, N-0316 Oslo, Norway

E-mail address: fredb@math.uio.no

$U R L:$ http://folk.uio.no/fredb/

(Pål Nicolai Henriksen), Department of Mathematics, University of Oslo, P.O. Box 1053, BLINDERn, N-0316 OsLO, NORWAY

E-mail address: paalnhe@math.uio.no 